\title{
Train Pipe State Detection Device Design
}

\author{
Shutao Wei
}

\begin{abstract}
Train pipe perforation state plays a crucial role on train safety. However, it is difficult to identify for complex sceneries and safety requirement. For the special design problem, in the paper, the reference parameter setting, the error decision, software filtering and interference suppression and other methods are all discussed to improve the measurement accuracy.
\end{abstract}

Index Terms-Perforation state, train pipe, reference parameter, negligence error, software filtering.

\section{INTRODUCTION}

At present, air brake is used for train brake, and sometimes negligence of workers, damage by misuse or non-human factors could result in the abnormal close of angular plug doors. Thereby it blocks the gas path, causes the brake system failure which leads to the train traffic accident happens, and brings the huge losses of lives and property. Especially since the 1990s, to improve the work efficiency of train operations and marshalling, the tail caboose has been removed, and the problem deteriorates. In recent years, under the auspices of the Ministry of Railways, a variety of anti-fold closing device and testing equipment have been developed. Though they can prevent knuckle cock abnormal close to some degree, from the aspects of technology, economics and management, they have their own limits and are hard to satisfy requirements, rapid and accurate measurement. Train Pipe State Detection device proposed in the paper can real-time detects the pipe perforation state at each brake operation which helps drivers to make the right judgment. Thus, it is very important for the protection of railway transport traffic safety and avoiding vicious accidents [1].

The measurement error problem in train pipe state detection device is dealt with in the paper. Several related factors about measurement device are analyzed, and the optimized device design scheme and parameter setting to improve the measurement accuracy are also discussed. Moreover, the negligent error deletion, software filter and system anti-disturbance and other design methods and techniques are also discussed. The measures have been used to the system design of detection device, and better results have been obtained.

\section{IMPACT FACTORS AND REFERENCE PARAMETER SETTING}

The design principle of train pipe state detection device is based the function between air pressure, flow and the number of vehicles on brake operation during running.

$$
N=f(Q, \Delta P, K)
$$

When the train is charging or reducing pressure to brake during running, the work gas is to reach every vehicle through the brake main pipe. If man-made or natural factors, the train angular cock is turned off at the No. $N$ locomotive section, then the charging and exhaustion air of brake main pipe $Q$ will change accordingly which is related to the charging and exhaustion pressure reduction amount $\Delta P$ enforced by drivers and different locomotives and brake devices, that is the instrument meter factor $K$ in equation (1). The total amount of gas in the system $Q$ is related to not only the pressure reduction amount $\Delta P$, but also working temperature $T$, air leak of brake pipe $V$, that is:

$$
d Q=K\left(\frac{\partial Q}{\partial N} d N+\frac{\partial Q}{\partial P} d F+\frac{\partial Q}{\partial T}+\frac{\partial Q}{\partial V}\right)
$$

As for the factors of the diameter, tubular and pipe roughness and other factors of vehicle itself are also included in the locomotive meter coefficient $K$. In fact, $Q, \Delta P$ and $N$ are non-linear relationship, however, under the same number of section $N$, the relation between $Q-\Delta P$ and $Q-N$ under the same $\Delta P$, are simulated by computer and experimented. The first order linear fit can satisfy the requirement of measure errors. The impact of working temperature can be compensated by hardware circuits, and its effects are better; however the impact of gas leak amount $V$ has great randomness.

It is difficult for the designer of train pipe state detection device to deal with random gas leak amount $\mathrm{V}$ and automatic air supplement operations, because when the train is with more train cars, the difference between the charging air and automatic air supplement of mitigation operation [2]. However, the maximum air leak has been set, since the air leak and supplement are hard to deal with, and the detection device utilizes the change amount of Q-P to measure when mitigation or brake operations, the air leak and charging can be amputated as relatively static parameters. Furthermore, the charging and exhaustion mechanism for the pipe air on the mitigation and brake operation are different respectively, so the device sets two reference values EH and EZ, and its amount of air leak and charging are both below these two values which can prevent the air leak impact on measurement to a maximum degree. If there are occasional false triggers, due to the reference value, the program can filter out in a timely manner.

\section{NEGLIGENT ERROR DETERMINATION AND REMOVAL}

Because of the on-site burst strong interference, there are
Shutao Wei is with the College of Computer and Information, Shanghai Second Polytechnic University, Shanghai, China (e-mail: chenl@ sspu.edu.cn). 
inevitable air leak when the train runs with more cars and the air surge impact of train pipe air flow under different road conditions, and the mistaken triggers caused by a variety of causes in the train running, the measure data list has inevitable negligent errors. It is an error that is obviously in consistent with the fact, is a serious distortion of the measurement results, and is the main reason for the low measurement accuracy of the detection equipment. Thus, it must be removed [3].

The negligent determination criterion generally uses the White rule with the measure numbers $n \rightarrow \infty$. It uses $3 \sigma$ as decision method, and has been widely applied to many computer processing software [4]. However, when $n$ is a limited value, it is obviously less reliable. If the $t$ distribution (student distribution) is adopted which is suitable for limited measurement, then the decision acquisition related with $\mathrm{t}$ and freedom degree $n$ has also a lot of problem in use. If the confidence probability is set to too small, the normal measurement data may be as abnormal data and deleted; otherwise, if it is too big, the abnormal data cannot be pick out. In the actual measurement, the pressure value $P$ of the brake pipe is less affected by various factors, and the measurement consistency of flow $Q$ is worse, and is easily affected by other factors. After careful analysis of the $P$ and $Q$ measurement data, the following categories of data are removed from the detection list [3].

1) The corresponding $P$ and $Q$ value when the pressure change amount $\Delta p<\frac{1}{2} \Delta p_{\min }$.

2) The corresponding $Q$ when the pressure change amount $\Delta p>\frac{1}{2} \Delta p_{\min }$ and $Q$ is greater than $Q_{\max }$.

3) The corresponding $P$ and $Q$ when the pressure change rate $P^{\prime}<P^{\prime}{ }_{\min }$.

4) The corresponding $P$ and $Q$ when the polarity consistency of pressure change rate $P^{\prime}$ is $<80 \%$.

where, $\Delta P_{\min }$ is the minimum pressure change of brake pipe with the minimum effective operation, and $Q_{\max }$ is the corresponding flow value in the maximum measurement scope with effective operation.

The above judgment rules are located in different positions of programs which can effectively remove the negligent error interference, increase greatly the measurement data reliability. The data dealt with by the above program can be considered as consistent effective measuring data, and they will be further processed by micro controller according to some algorithm rules.

\section{Software Filtering}

This kind of complex system having a variety of non-ideal factors, the signal bond detected by device has great randomness, and its characteristics can only be described by statistics. The traditional filtering method is impossible to separate unwanted signal from the measure data list. Therefore, to obtain a higher credits useful data, the software algorithm extracting real data is adopted. Furthermore, because its strong self-adaptability, its effect is beyond the reach of hardware filter circuit [5]. Of course, taking up a lot of CPU running time is its fatal weakness. Whether or not the software filtering is added into system is entirely dependent on the frequency of signal change, A/D conversion rate and reasonable selection of sample numbers and other factors.

For $n$ numbers of data collection, there is negligent error caused by system error, random error and burst interference which all deviate data from the true value. At this time, the $\mathrm{n}-\mathrm{m}$ measure data arithmetic mean value $\bar{y}_{\text {is }}$ as the true value of measurement results excluding $m$ error data.

$$
\bar{y}=\frac{1}{n-m} \sum_{i=1}^{n=m} y i
$$

where, $y i$ is the ith system parameter measurement value.

The arithmetic mean value $\bar{y}$ of limited numbers of measurement sequences replaces the true value $y$ which applies the arithmetic mean principle[5]. The value of $N$ is determined by system characteristics and complies with Bessel root mean square error formula.

$$
\begin{gathered}
\lambda=\sqrt{\frac{1}{n-m-1} \sum_{i=1}^{n-m}[y i-\bar{y}]^{2}} \\
=\sqrt{\frac{1}{n-m-1} \sum_{i=1}^{n-m}[\Delta y i]^{2}}=\sqrt{\frac{\varepsilon \min }{n-m-1}}
\end{gathered}
$$

The formula not only illustrates the error square sum $\varepsilon$ that the arithmetic mean value replaces the true value is the smallest, and the more numbers of data collection, the smaller the value of $\lambda$ is. But, $\lambda$ reduces according to the rate of $\sqrt{n-m-1}$, so the reduced rate of $\lambda$ is much slower than $n-m$. After n-m.>10, the reduced effect is not obvious. Moreover, when $\mathrm{n}$ is too large, the equal precision measurement condition does not exist. Thus, $n-m=4 \sim 10$ is more suitable. In the paper, the proposed detection device considers the value as 8 according to the measurement accuracy requirement and speed and other factors. The software filter method is very effective for enhancing the measurement accuracy of device.

\section{SYSTEM ANTI-INTERFERENCE}

To improve the system anti-interference performance, avoid strong external electromagnetic interference on the weak signal, in the detection device the sensor and signal conditioning board are placed on the same detection box, and shorten their connection line as much as possible. The detection box is directly connected in series in the train brake pipe. The signal conditioning board sends the amplified current signal. This current signal is not sensitive to the change of the excitation voltage, long wire transmission error will not be generated for voltage drop or induced noise which enhances greatly its anti-interference ability. The 4-core cable is used to connect the measuring box and host which effectively prevents the space of electromagnetic waves from the external input [6].

In the experiment, it is discovered that despite the use of such strict preventive measures, the measurement 
In the experiment, it is discovered that despite the use of such strict preventive measures, the measurement performance in remote areas is still better than in big cities and busy stations. This also shows that the electromagnetic pollution in the locomotive is more serious, and the electromagnetic shielding performance of instrument is not perfect.

The most important and serious interference of the general computer detection system is the power pollution. It is estimated that more than $90 \%$ of computer failures is caused by power supply noise [6]. Computer detection system generally has a large load work equipment, so the power supply pollution problem should be pay full attention. In the design, the independent, distribution, dispersed and function block supply principle is adopted. The analog and digital module power and ground lines are not only strictly separated, but also the display circuit and other large load parts are supplied by independent power which reduces external influences on micro processors as much as possible, and improve the power supply reliability to some extent. When train climbs with great load, the motorcycle meter indicates some power currents may reach several hundred amperes that are several times than in the straight road which causes electric and magnetic changes, and then has impacts on the detection devices' normal works. Thus, later, the anti-surge technology has been used in the power supply design which mitigates them.

\section{CONCLUSION}

In the paper, the reference parameter setting, negligent error, software filter and system anti-interference methods are deal with which are intelligent design principle based on micro control unit. MCU and high speed A/D are introduced to provide better conditions for pipe state detection device data collection and process which is a good solution to the mitigation and braking process bidirectional accurate detection, and decompression amount arbitrary during operation.

The detection device based on the proposed principle has been developed. The optimum scope of reference value is determined by on-site dynamic and static train load experiments. Furthermore, the algorithmic average value of $n-m$ collections with removal of negligent errors is adapted to as real measurement value to participate calculation. It can provide the accurate position information about angular cock abnormal shut or exceptional block. Moreover, the corresponding position is represented by the number of locomotive cars, the measurement errors $\leq \pm 3$ sections which satisfies the requirements of train actual operation. In recent ten years, this detection device is tested by small-scale and pilot experiments of train sector. After continuous improvement, it has finally into the electrical equipment box and plays its due role in the transport of railway safety.

\section{REFERENCES}

[1] S. T. Wei, J. C. Xu, Y. Q. Sun et al., "Design of a Intelligent monitoring instrument of breakthrough status in train," Microelectronics \& Computer, vol. 17, no. 5, pp. 15-19, 2000.

[2] Z. Y. Ma, "Using locomotive supervise and record apparatus to judge the condition of train line angle cock," Diesel Locomotives, vol. 4, pp. 54-57, 2004.

[3] T. Zhang and B. Wang, "New judgment method of continuous outliers," Journal of Southeast University, vol. 35, Sup (II), pp. 225-227, 2005.

[4] X. J. Jin, "Outliers elimination method of ballistic measurement data based on least square," Sichuan Ordnance Journal, vol. 32, no. 1, pp. 20-23, Jan. 2011.

[5] H. W. Jiang and X. Sun, Electrical Measuring, 1st ed. Chichester, Beijing: China Metrology Publishing House, 1988.

[6] K. R. Xiong and J. Ni, "Detection instrument dedicated microcontroller system design," Computer Engineering and Applications, vol. 33, no. 8, pp. 64-66, 1997.

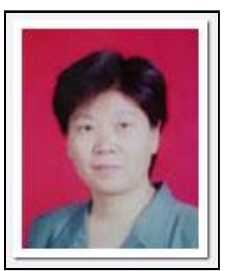

Shutao Wei was born in Henan Province, China, in 1954. He received the B.S. degree from the Tsinghua University, Beijing, in 1977 in communication engineering. She is currently a professor with the College of Computer and Information, Shanghai Second Polytechnic University. His research interests include signal detection, embedded system. 